\section{Lentes de contacto y cálculo de lente intraocular: sorpresa refractiva usando el método biométrico de no contacto IOLMaster}

\section{Contact lenses and intraocular lens power calculation: refractive surprise using the IOLMaster non-contact biometric method}

\section{Sr. Director:}

El objetivo de la presente es alertar del peligro de realizar el cálculo biométrico de la lente intraocular, a pesar de utilizar la última tecnología de máxima precisión, desconociendo que el paciente lleve colocada la lente de contacto en ese momento.

El error en el cálculo de la lente intraocular (LIO) es actualmente la causa más frecuente tanto de recambio de la lente como de reclamaciones legales (1). El desarrollo de la biometría de no contacto con interferometría láser (IOL-MASTER, Zeiss) ha permitido aumentar la exactitud de dicho cálculo, especialmente importante en la cirugía refractiva de cristalino transparente, donde el objetivo prioritariamente es la emetropía postoperatoria.

Presentamos un caso de una paciente de 58 años con opacidad de cristalino incipiente y visión 10/10 con refracción de $-8,25$ en ambos ojos, que fue programada para cirugía intraocular y lente multifocal. En el intervalo entre la exploración oftalmológica y la toma de medidas por el personal técnico auxiliar de enfermería y óptico-optometrista, sin consulta previa ni posibilidad de ser detectado por el equipo médico, la paciente se colocó sus lentes de contacto, realizándose con ellas la medida biométrica. Dicho cálculo biométrico fue realizado mediante el sistema Zeiss IOLMaster (V.3.01; Carl Zeiss, Jena, Germany).

La longitud axial y queratometría media determinada fue, en OD, de 26,21 mm/37,42 dioptrías corneales (D) y en OI de $26,03 \mathrm{~mm} / 38,63 \mathrm{D}$, lo que estableció un cálculo para emetropía de la lente intraocular multifocal (366D Acri.tec, constante $\mathrm{A}=118,3$ ), con la fórmula de Haigis de +21 OD y +19 OI. Tras la cirugía en OD la agudeza visual sin corrección era menor de $1 / 10$ con una graduación de $-7,25$. Repetido el cálculo biométrico en el ojo contralateral (OI) volvió a dar el mismo resultado $(+19,5)$, pero al percibir que portaba la lente de contacto $(-7 \mathrm{D}$, radio 8,6 , Purevision, Bausch\&Lomb), procedimos a su retirada y repetimos el cálculo. Los nuevos datos fueron: OD: $26,14 \mathrm{~mm} / 44,77 \mathrm{D}$; OI: $26,03 \mathrm{~mm} / 45,08 \mathrm{D}$. Tras recambio de la LIO en OD y cirugía con LIO ajustada en potencia en OI la graduación final fue de OD: $-0,25-0,25$ a $20^{\circ}$, OI: $-0,5$ emetropía bilateral satisfactoria sin necesidad de gafas.

Es conocida la influencia de las lentes de contacto en poder modificar el cálculo biométrico de la lente intraocular, especialmente las duras y permeables al gas al modificar la curvatura corneal y por tanto las dioptrías corneales $(\mathrm{K})$, a veces hasta semanas después de su retirada (2), pero revisado PubMed en la literatura mundial sólo existe un caso similar al presente de error biométrico grave por realizar la biometría con lente de contacto blanda desechable puesta (3).

Concluimos que a pesar de utilizar la última tecnología en el cálculo de las lentes intraoculares (IOLMASTER), es muy importante cerciorarse de que el paciente no haya colocado o porte la lente de contacto, aunque sea blanda y desechable, para evitar sorpresas refractivas que pueden llegar a ser de hasta siete dioptrías, como observamos en el presente caso.

Díaz-Llopis $\mathrm{M}^{1}$, García-Delpech $\mathrm{S}^{1}$, Quijada $\mathrm{A}^{2}$, Salom $\mathrm{D}^{2}$, Udaondo $\mathrm{P}^{2}$

1 Doctor en Medicina.

2 Licenciado en Medicina. Hospital General Universitario de Valencia. Universitat de Valencia. España E-mail: garcia@iova.es

\section{BIBLIOGRAFÍA}

1. Mamalis N. Complications of foldable intraocular lenses requiring explantation or secondary intervention-2001 survey update. J Cataract Refract Surg 2002; 28: 2193 2201.

2. Cleasby GW, Dadson A. The effects of hard contact lenses on intraocular lens calculations. American Intra-Ocular Implant Society 1985; 11: 603-604.

3. Nguyen DQ, Hakin KN. Contact Lens Wear and IOL Power Calculation Before Cataract Surgery: A cautionary tale. J Refract Surg 2006; 22: 204. 